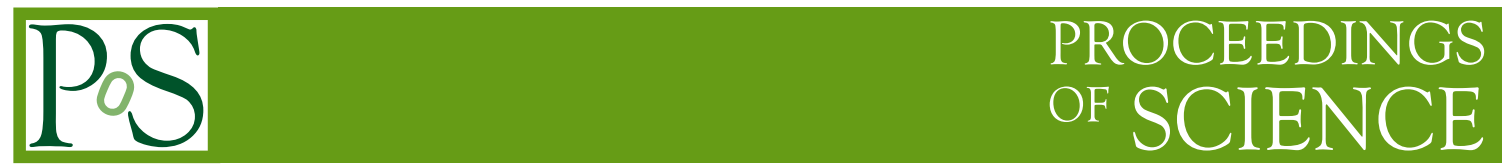

\title{
Central exclusive meson production at $\mathrm{LHCb}$
}

\author{
Paolo Gandini* \\ University of Oxford \\ on behalf of the LHCb Collaboration \\ E-mail: paolo.gandini@cern.ch
}

The forward acceptance of the $\mathrm{LHCb}$ detector provides a unique opportunity for the measurement of QCD phenomena at large rapidities and low transverse momenta, where theoretical models often fail to describe the data accurately. We present new exclusive production studies of $\Upsilon$ mesons. A first look at the new Run II data and the performance of the new HERSCHEL forward counters are presented.

The European Physical Society Conference on High Energy Physics 22-29 July 2015

Vienna, Austria

${ }^{*}$ Speaker. 


\section{Introduction}

The LHCb detector [1] has proven to be a well suited detector for Central Exclusive Production (CEP) studies [2, 3, 4]. CEP events involve proton-proton interactions of the form $p p \rightarrow p+X+p$, where the two protons remain intact. $X$ is the central exclusively produced object (reconstructed inside the acceptance of the detector), while + signs indicate a rapidity gap. CEP processes are mediated by photon-pomeron, pomeron-pomeron and photon-photon exchanges. LHCb provides a clean experimental environment to detect the low- $p_{T}$ objects produced. Such processes can be calculated perturbatively and are sensitive to the gluon parton density function down to Bjorken$x$ of $1.5 \times 10^{-5}$ in detector. CEP events are triggered in LHCb by requiring low activity in the detector (trackers and calorimeters). The size of the rapidity gaps is critical to the characterisation of the events. The typical topology of a CEP event in LHCb is shown in Figure 1.
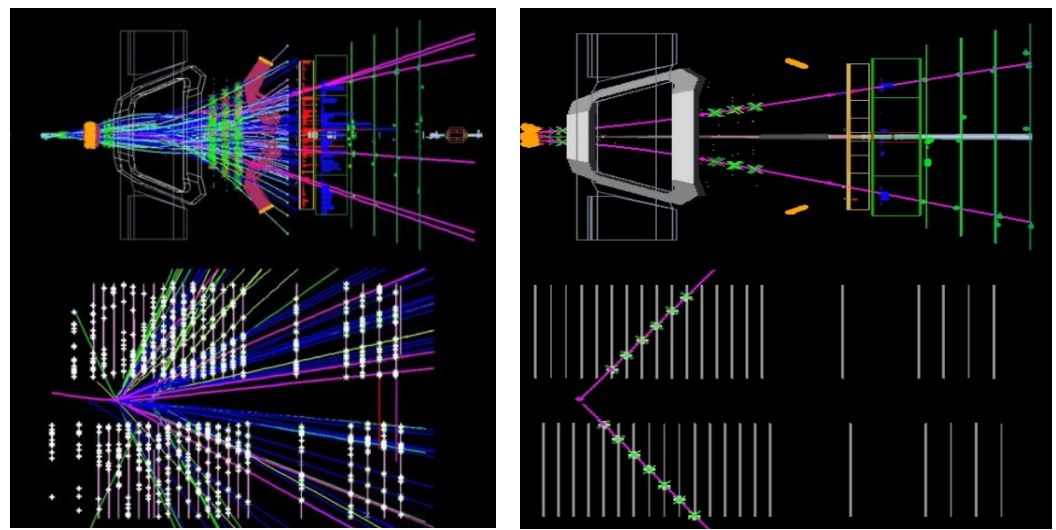

Figure 1: Topology of a typical LHCb event (left) and a CEP-like event (right). The image shows a top view of the detector and a zoom of the Vertex Locator (VELO). The vertical grey lines represent the detection planes of the VELO.

\section{Exclusive $\Upsilon$ production at $\mathrm{LHCb}$}

The CEP of $\Upsilon(1 S), \Upsilon(2 S)$ and $\Upsilon(3 S)$ mesons is measured at LHCb [4] requiring two wellreconstructed muons and little other activity in the detector. Backgrounds are identified and quantified by data-driven procedures. The background from feeddown from $\chi_{b} \rightarrow \Upsilon \gamma$ is estimated by reconstructing this decay from $\Upsilon$ photon candidates in the data and correcting for the efficiency estimated from the simulation. The fraction of non-exclusive $\Upsilon$ production, where the proton remnants stay outside the $\mathrm{LHCb}$ acceptance and are thus not detected, is statistically separated from the signal by fitting the $p_{T}^{2}$ distribution of the signal candidates (Figure 2). Signal templates are derived using the SuperChiC event generator [5]. The measured production cross-section for the $\Upsilon(1 S)$ and the resulting photo-production cross-section are shown in Figure 3. A good agreement is found between data and the perturbative predictions made at next-to-leading order (NLO) [6]. 

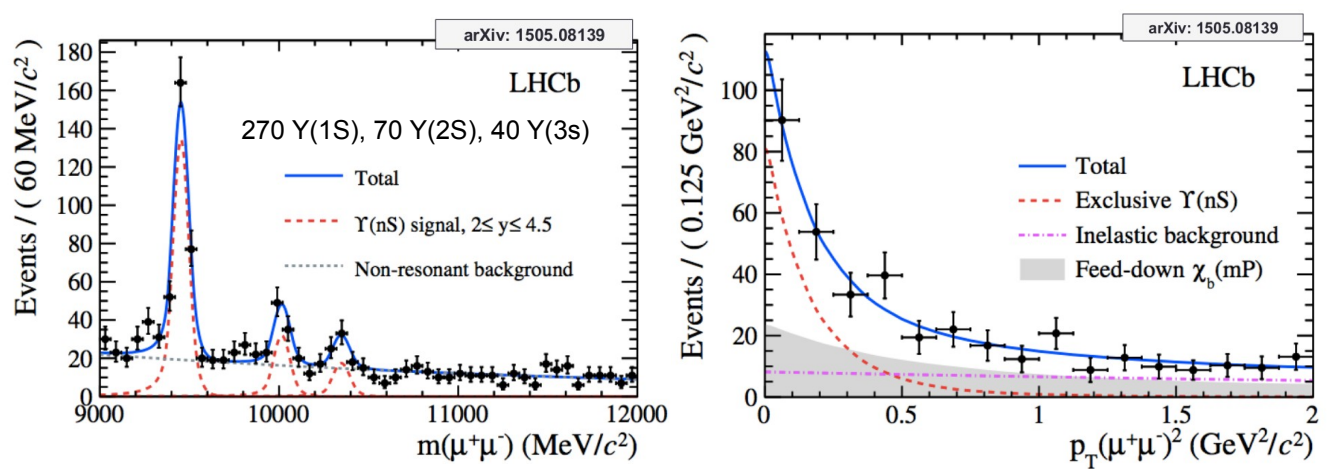

Figure 2: $\Upsilon(1 S), \Upsilon(2 S)$ and $\Upsilon(3 S)$ mass distribution for CEP events (left). Fit to the $p_{T}^{2}$ distribution of the $\Upsilon(1 S)$ meson (right).
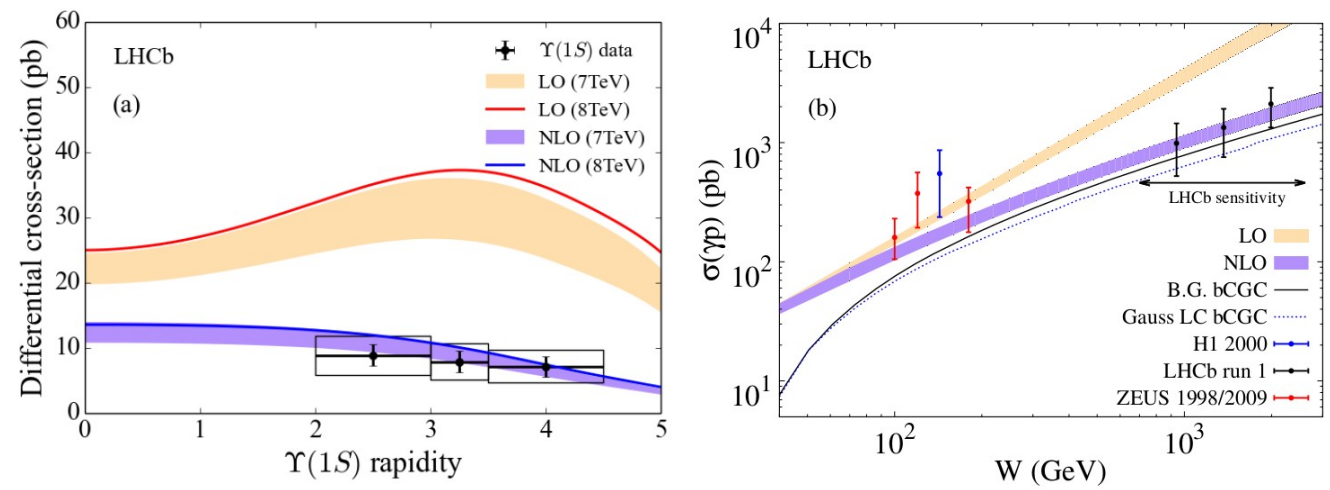

Figure 3: Differential cross-section as a function of rapidity, measured for the $\Upsilon(1 S)$ (left). Derived photoproduction cross-section as a function of photon-proton interaction energy [6] (right). The kinematic region probed with $\mathrm{LHCb}$ is indicated with the double-headed arrow.

\section{The Herschel detector}

CEP analyses benefit from an increased pseudorapidity $(\eta)$ coverage of the detector. The ability to identify larger rapidity gaps in candidate events is beneficial to studies of exclusive production, therefore a new sub-detector has been installed in LHCb for Run-II data taking. High Rapidity Shower Counters for LHCb (Herschel) [7] have been installed in the LHC tunnel, where space was available either side of interaction point 8 . The Herschel detector comprises five planes of $20 \mathrm{~mm}$ thick scintillators, each divided into four quadrants and using the same electronics as the $\mathrm{LHCb}$ pre-shower detector. It provides acceptance coverage up to $\eta=8$ and it can be used to veto forward and backward activity. The layout of the detector is shown in Figure 4. Fully commissioned before data-taking, the HeRSCheL detector has been fully integrated in the LHCb DAQ and it is being run together with the rest of the experiment. Work is ongoing to integrate Herschel at the hardware trigger level (L0), which could provide optimal background rejection while improving trigger efficiency in Run-II. 


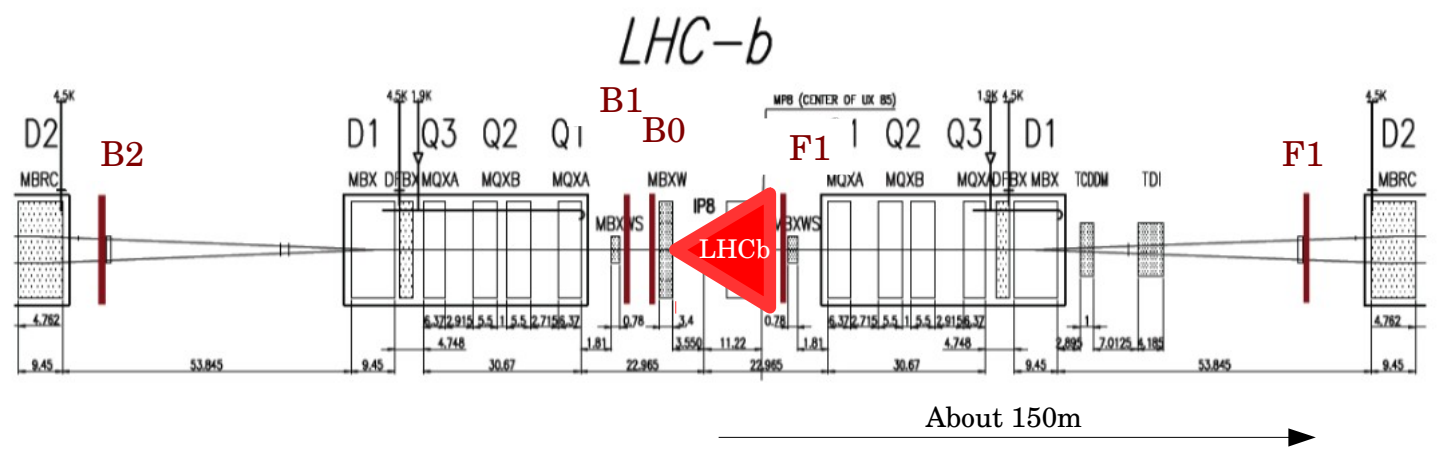

Figure 4: Layout of the Herschel detector. The LHCb main detector is sketched as a red triangle. The five stations B0,B1 (backward) and F0,F1,F2 (forward) are drawn in red.

\section{A first look at Run-II data (13TeV $p p$ collisions)}

The LHC was scheduled to run a luminosity ramp up period with $50 \mathrm{~ns}$ bunch spacing before moving to "nominal" $25 \mathrm{~ns}$ spacing conditions. LHCb has used the ramp up period to collect luminosity with dedicated loose triggers, including low-multiplicity triggers. We report preliminary studies of a dataset collected during this period, corresponding to an integrated luminosity of about $5 \mathrm{pb}^{-1}$. Dedicated low-multiplicity triggers have been designed to select di-hadron, di-muon and di-photon modes with high efficiency. An example of a di-pion candidate produced in a lowmultiplicity $13 \mathrm{TeV} p p$ collision is shown in Figure 5, where two tracks consistent with the pion hypothesis (PID is applied) are reconstructed in the acceptance of LHCb and little other activity is recorded in the detector. The benchmark channel $J / \Psi \rightarrow \mu^{+} \mu^{-}$is also reconstructed in the $50 \mathrm{~ns}$
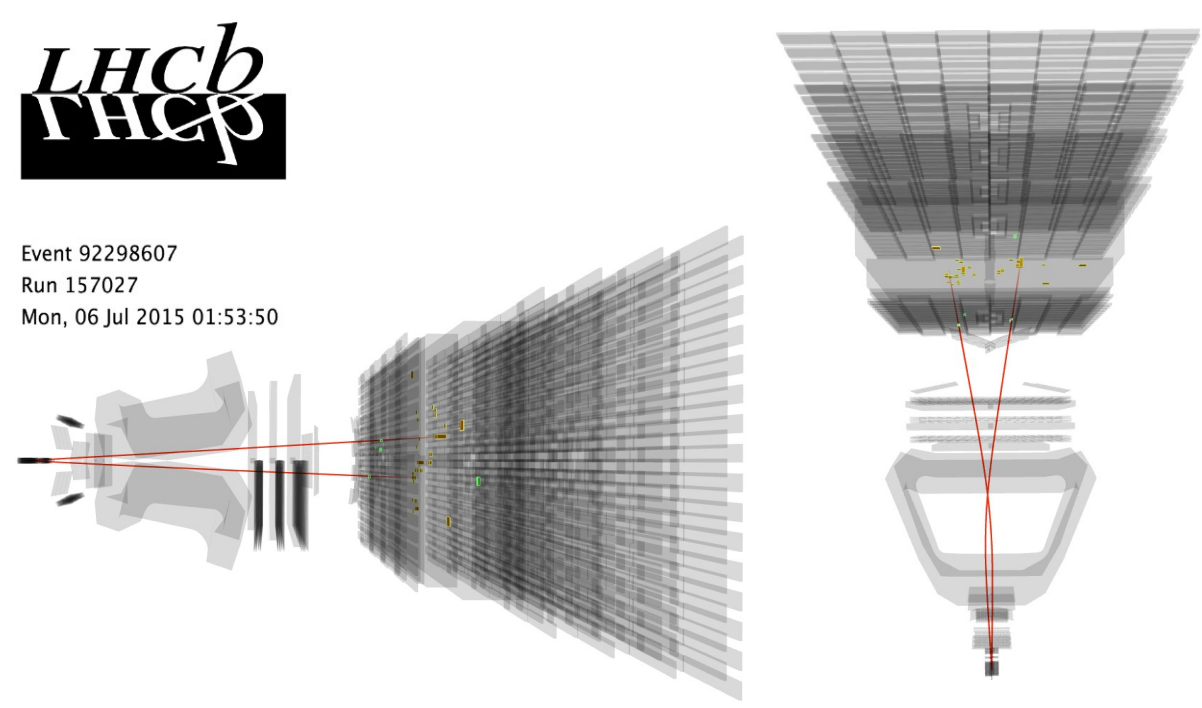

Figure 5: Di-pion event collected in July 2015. Two tracks are reconstructed with little other activity in the detector.

data at $13 \mathrm{TeV}$ to look for di-muon events consistent with CEP. Di-muon candidates are selected by 
requiring two good muon tracks with loose muon PID criteria applied, no other tracks reconstructed in the detector and no photons in the calorimeter. The preshower detector is also required to have low activity, a requirement useful in the L0 hardware trigger. Clear signals of exclusive-like $J / \Psi$ and $\Psi(2 S)$ are shown in Figure 6.

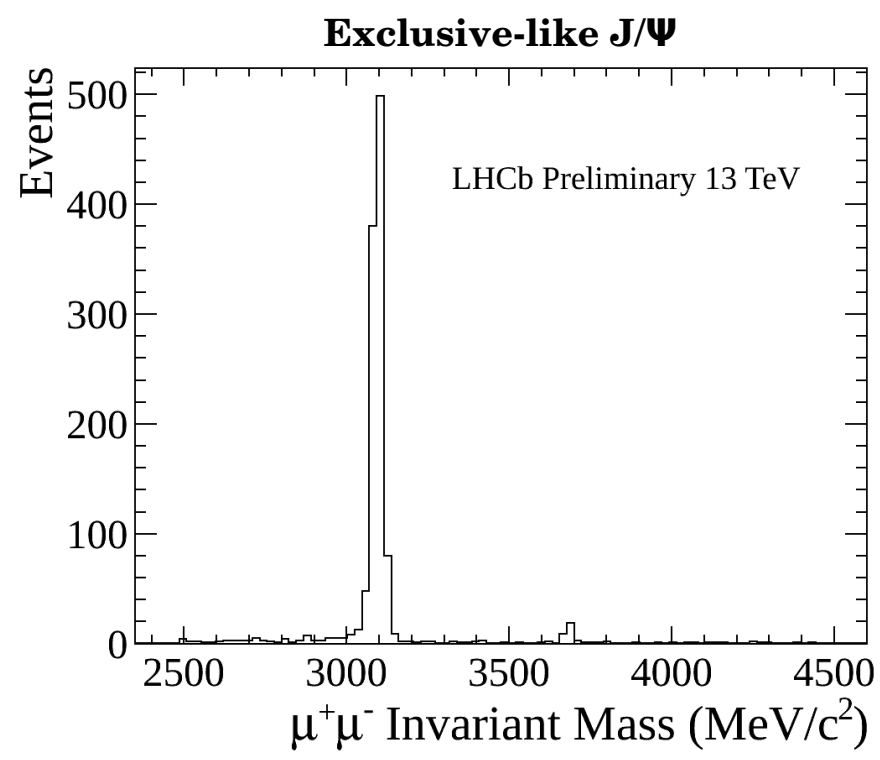

Figure 6: Di-muon events collected in summer 2015 at $13 \mathrm{TeV}$ in the mass region of the $J / \Psi$, once lowmultiplicity and exclusivity cuts are applied (integrated luminosity $5 \mathrm{pb}^{-1}$ ).

\section{Conclusions}

The LHCb CEP program is quickly expanding. With charmonium [2], double-charmonium [3] and bottomonium [4] results published so far using Run-I data, new analyses are expected using both Run-I and Run-II datasets. New forward shower counters have been installed and are being successfully operated in 2015. They will allow good rejection of diffractive backgrounds in future CEP analyses. $\mathrm{LHCb}$ is scheduled to collect more than $200 \mathrm{pb}^{-1}$ of data in 2015 (at the time of writing the collected integrated luminosity exceeds $300 \mathrm{pb}^{-1}$ ), in which loose dedicated lowmultiplicity triggers are included.

\section{References}

[1] Alves, Jr., A. and others, The LHCb Detector at the LHC, JINST, 3 (2008) S08005

[2] Aaij, R. and others, Updated measurements of exclusive $J / \Psi$ and $\Psi(2 S)$ production cross-sections in $p p$ collisions at $\sqrt{s}=7 \mathrm{TeV}$, J. Phys. G. 41 (2014) 055002

[3] Aaij, R. and others, Observation of charmonium pairs produced exclusively in $p p$ collisions, J. Phys. G. 41 (2014) 115002 
[4] Aaij, R. and others, Measurement of the exclusive $\Upsilon$ production cross-section in pp collisions at $\sqrt{s}=7 \mathrm{TeV}$ and $8 \mathrm{TeV}$, JHEP 1509 (2015) 084

[5] Harland-Lang L. A. and others, Standard candle central exclusive processes at the Tevatron and LHC, EPJC 69 (2010) 179

[6] Jones, S. P. and others, Probes of the small $x$ gluon via exclusive $J / \psi$ and $\Upsilon$ production at HERA and the LHC, JHEP 11 (2013) 085

[7] FSC Team (CMS), HERSCHEL Team (LHCb), M. Albrow, P. Collins, and A. Penzo, J. Mod. Phys. A 29 (2014) 1446018 